\title{
COMPARATIVE EFFECT OF FLAXSEED OIL AND FISH OIL IN ACETIC ACID- INDUCED COLITIS IN RATS
}

Aisha Dugani ${ }^{1}$, Ahlam Elhelawi ${ }^{1}$, Aisha Edrah ${ }^{2}$, 1) Department of Pharmacology and Clinical Pharmacy, University of Tripoli, Tripoli, Libya, 2) Department of Pharmacology, Faculty of Medicine, Tripoli University, Tripoli, Libya.

Received: 26 April 2012, Accepted in revised form: 18 Jun 2012, Published: 7 July 2012

Corresponding author: Aisha Dugani, e- mail: duganiaisha@yahoo.com

\section{ABSTRACT:}

Objectives: The aim of the present study was to evaluate the possible protective effect of flaxseed oil and to compare this effect with fish oil in experimental ulcerative colitis (UC).

Methods: Rats were equally divided into five groups of six animals each. Sham control group (corn oil, $1 \mathrm{ml}$ ), acetic acid group (normal saline, $1 \mathrm{ml}$ ), flaxseed oil group (FSO, $1 \mathrm{ml}$ ), fish oil group (FO, $1 \mathrm{ml}$ ) and mesalamine-treated group ( $3 \mathrm{ml})$ as a positive control. All drugs were administered intrarectally (IR). One hour following treatment in the acetic acid group, FSO group, FO group and mesalamine group, $1 \mathrm{ml}$ of $4 \%$ acetic acid was introduced as an enema. Rats were sacrificed after $24 \mathrm{hrs}$ and histopathological scores of the all colonic specimens were assessed by microscope. Colonic weight/length ratio was also evaluated.

Results: Microscopical improvement as manifested by the reduction in the inflammatory score and normalization of intestinal mucosal architecture was observed in fish oil pretreated rats compared to acetic acid group but there was no significant difference in flaxseed oil pretreated group. The decrease in weight/length ratio was statistically significant in fish oil-treated group compared with acetic acid group, but there was no significant difference between flaxseed oil-treated and acetic acid group.

Conclusion: The results of this study suggest that fish oil but not flaxseed oil could ameliorate the mucosal damage in experimentally induced ulcerative colitis in rats when given in the form of an enema.

Keywords: Flaxseed oil; ulcerative colitis; fish oil; acetic acid induced colitis.

\section{الملخص العقربِي:

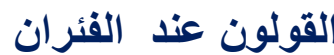

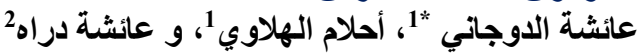

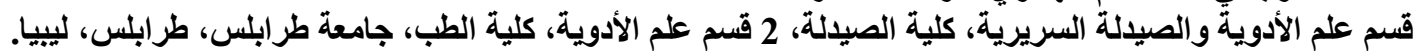

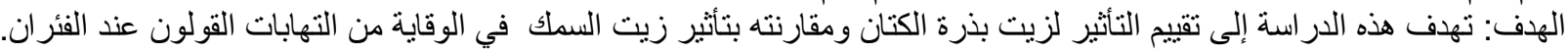

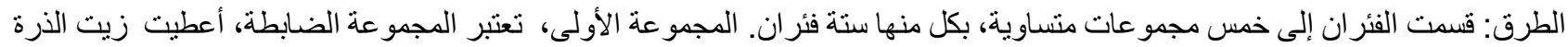

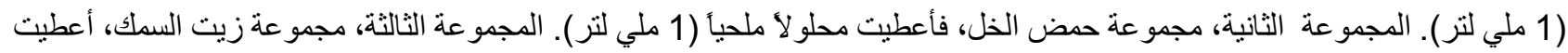

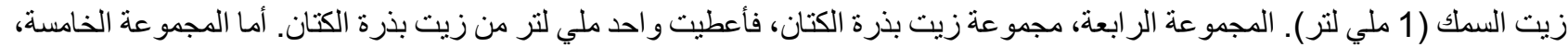

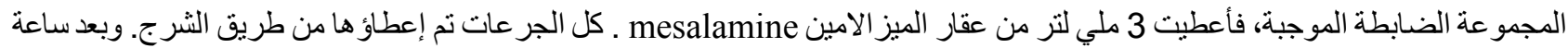

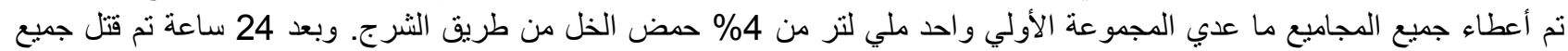

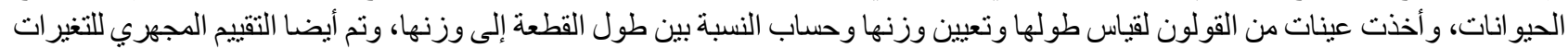

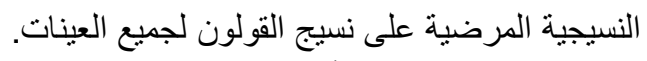

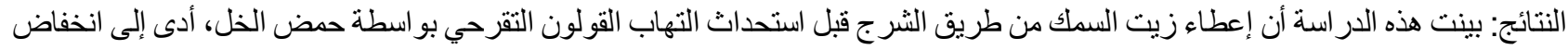

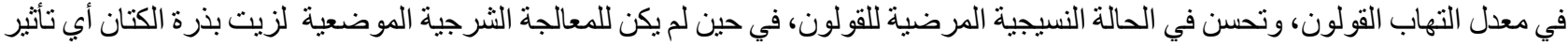

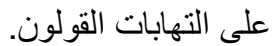
الاستتاجات: بينت نتائج هذه الدر اسة أن حقن زيت السمك، وليس زيت بذرة الكتان الكان من طريق الثرج له تأثير جيد في التقليل من التخرشات التي

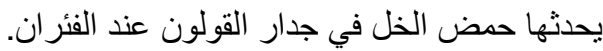
الكلمات مفتاحية: زيت بذرة الكتان، زيت السمك، التهاب القولون التقرحي، حمض الخل. 
INTRODUCTION:

T Tcerative colitis (UC) is a form of inflammatory bowel disease (IBD) characterized by diffuse mucosal inflammation of the colon and rectum and typically involves only the innermost lining of the mucosa, manifesting as continuous areas of inflammation and ulceration with no segments of normal tissue [1]. The disease typically starts in the rectum, but often extends to involve the whole length of the colon [2].Despite the enormous research on the pathogenesis of UC, the exact cause of the condition remains not completely understood. The disease appears to be related to combination of genetic and environmental factors [1].The pathological findings associated with UC include: an increase in inflammatory mediators such as prostaglandins (PG) and leukotrienes (LT), which are produced from arachidonic acid. Clinical and experimental studies have confirmed that the levels of prostaglandin E2, thromboxane A2, prostacyclin, and especially LTB4 during colonic inflammation were highly increased [3]. Other factors include: oxidative stress, altered colonic milieu, abnormal mucosal content of glycosaminoglycan (GAG), decreased oxidation of short chain fatty acids (SCFAs), increased intestinal permeability, increased sulfide production, and decreased methylation [1].Treatment of IBD including UC consists of sulphasalazine (SSZ), 5-aminosalicylic acid (5ASA), corticosteroids, immunomodulator drugs [azathioprine (AZA) and methotrexate (MTX)], 6 mercaptopurine (6-MP), calcineurin inhibitors (cyclosporin and tacrolimus), and anti-TNF-alpha antibodies (infliximab, adalimumab and certolizumab). The choice of treatment depends on the clinical goal (induction or maintenance of remission), extent and severity of disease, response to current or prior medication and the presence of complications [4].Polyunsaturated fatty acids (FA) of the omega-3 class, especially eicosapentaenoic acid (EPA) and docosahexaenoic acid (DHA) have attracted interest in recent years because of their anti-inflammatory properties [5]. Several published research work have confirmed the protective effect of fish oil supplemented in diet or as enema in experimental induced colitis [5-8] and also in clinical trials on patients with IBD [9-13]. Thus it was thought worthwhile to investigate the effect of other omega-3 rich oil such as flaxseed oil and to study the comparative effects of these

two oils in the protection against acetic acid inducing colitis in rats.

\section{MATERIALS AND METHODS: ANIMALS.}

Healthy Albino Wistar rats of either sex weighing 200-250 g were used in this study. They were randomly allocated to groups of 6 rats each. The animals were housed in the animal care facility in the department of Pharmacology and Clinical Pharmacy and maintained at $23^{\circ} \mathrm{C}$ with a 12 hour light and 12 hour dark cycle. All rats were fasted for 24 hours prior to the experimental procedure. The study was approved by the Faculty of Pharmacy and the experiments were done according to the ethics guidelines of Tripoli University.

\section{INDUCTION OF COLITIS AND TREATMENT PROTOCOL:}

Colitis was induced in rats by intrarectal (IR) administration of acetic acid (AA). After anesthetized with ketamine in a dose of 75 $\mathrm{mg} / \mathrm{kg}$ injected intraperitoneally, a soft $6 \mathrm{~F}$ polypropylene catheter lubricated with jelly was inserted $6-8 \mathrm{~cm}$ via the anal canal into the colon. One $\mathrm{ml}$ of $\mathrm{AA}(4 \%$ vol./vol. in $0.9 \%$ saline) was slowly infused into the distal colon and rats were maintained in a head-down position for 30 seconds to limit the expulsion of the solution [5].

\section{TREATMENT PROTOCOLS:}

Rats were randomized into five groups. Group 1: Sham control group (SC). One $\mathrm{ml}$ of normal saline was given IR after 1 hour from administration of one $\mathrm{ml}$ corn oil enema. Group 2: Control colitis group (CC), one $\mathrm{ml}$ of $4 \% \mathrm{AA}$ was administered IR 1 hour following IR administration of normal saline. Group 3: Fish oil treatment group (FO-IR), one $\mathrm{ml}$ of FO was administered as enema one hour before induction of colitis. Group 4: Flaxseed oil treatment group (FSO-IR), one $\mathrm{ml}$ of FSO was administered as enema one hour before induction of colitis. group 5: mesalazine treatment group, the animals were given $3 \mathrm{ml}$ of mesalazine enema one hour before induction of colitis.One hour following the above treatments, colitis was induced in all animal in groups 3,4 and 5 by the administration of AA IR $(1.0 \mathrm{ml}$ of $4 \%)$. All rats were scarified 24 hours following AA-treatment. 
ASSESSMENT OF COLONIC DAMAGE:

After midline laparotomy, the colon was removed, cleaned from fat and mesentery, and blotted on a filter paper. Each specimen was weighed and its length measured under a constant weight $(2 \mathrm{~g})$ and expressed as colon weight $(\mathrm{mg}) /$ length $(\mathrm{cm})$ ratio. The distal 2$3 \mathrm{~cm}$ segment of the colon was isolated from the rest of the tissue samples and opened longitudinally, rinsed with saline to remove luminal content and fixed in $10 \%$ formalin and embedded in paraffin, and $5 \mu \mathrm{m}$ sections were prepared. Tissues were routinely stained with hematoxylin and eosin and were evaluated for the appearance of muscle layers by a light microscopy by a pathologist who was not informed about the treatment protocols. The degree of colonic inflammation was evaluated by histologic scoring (from 0 to 3 ) as previously describe [14], where normal histologic appearance $=0 ; 1=$ inflammation on mucosa and submucosa; $2=$ inflammation on entire wall of the bowel, and $3=$ ulcer and necrosis of the entire wall.

\section{STATISTICAL ANALYSIS:}

Data are expressed as mean + S.E.M. Statistical calculations were done with SPSS 11.0 software package. Comparison between two groups was performed using Student's t-test and comparison between more than two groups was carried using one-way analysis of variance (ANOVA). Differences were considered significant when the degree of confidence was $95 \%$ or better $(\mathrm{P}<0.05)$.

\section{RESULTS:}

The intracolonic administration of $1.0 \mathrm{ml}$ of $4 \%$ AA produced a severe and significant inflammatory response in rat colon $24 \mathrm{hrs}$ after injection. This response is evidenced by the significant increase in weight of $5 \mathrm{~cm}$ distal colonic segment and an increase in the histopathological score as compared to control group treated with normal saline (Figure 1 and Figure 2B). All rats in the acetic acid treatment group developed diarrhea, in some animals bloody diarrhea was observed.

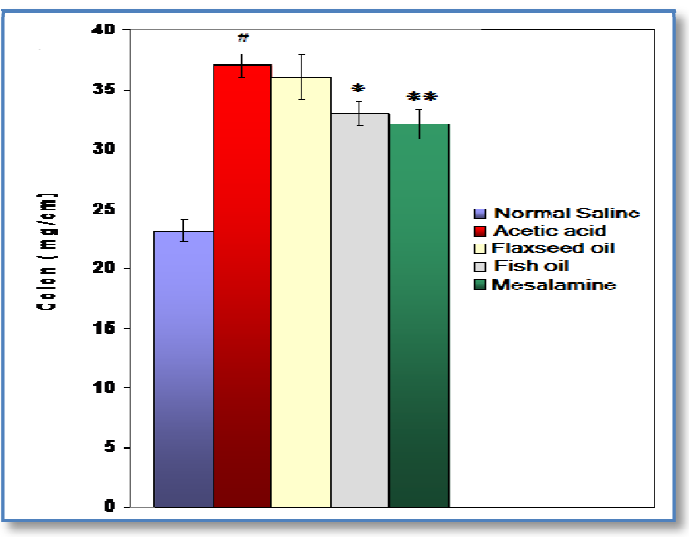

Figure1. Effect of pretreatment with fish oil, flaxseed oil and mesalamine on colon weight/length ratio in acetic acid- induced colitis in rats. Values are mean \pm $\mathrm{SEM}, \mathrm{n}=6 . \#, \mathrm{P}<0.001$ significantly higher than sham control (normal saline-treated); ${ }^{*}, \mathrm{P}<0.05$ and ${ }^{* *}, \mathrm{P}<0.01$ significantly lower from colitis group (acetic acidtreated group) respectively.

\section{EFFECTS OF FISH OIL AND FLAXSEED OIL ON COLONIC WEIGHT/ LENGTH RATIO IN ACETIC ACID- INDUCED COLITIS.}

The colonic tissue segment weight/length ratio was significantly higher in the AAgroup as compared to $\mathrm{SC}$ group $(37.0 \pm 1.0$ vs $23.12 \pm 0.9$ respectively, $\mathrm{P}<0.001$, Figure 1. Pretreatment of rats with one $\mathrm{ml}$ of FOIR, showed a significant mucosal protection effect against AA-induced colitis as evidenced by the decrease in the colon weight/length ratio in comparison with SCtreatment groups $(32.97 \pm 1.02$ vs $37.00 \pm$ $1.01, \mathrm{P}<0.05$ ). FSO pretreatment, on the other hand, has a slight but insignificant reduction in the colonic tissue weight/length ratio in comparison with AA- control group $(36.00 \pm 1.9$ vs $37.00 \pm 1.01$ respectively Figure 1.

\section{EFFECT OF FISH OIL AND FLAXSEED OIL ON COLONIC HISTOLOGICAL DAMAGE INDUCED BY ACETIC ACID.}

Representative histological segments of the colon in the control, AA- treatment group and in group of rats treated with FO, FSO and mesalamine enemas are shown in figure 2. AA produced a significant inflammatory response and prominent lesions in the mucosa and submucosa, with invasion of inflammatory cells mainly macrophages and neutrophils and dilation of capillaries and lymphatics, Figures 2B and 3. 


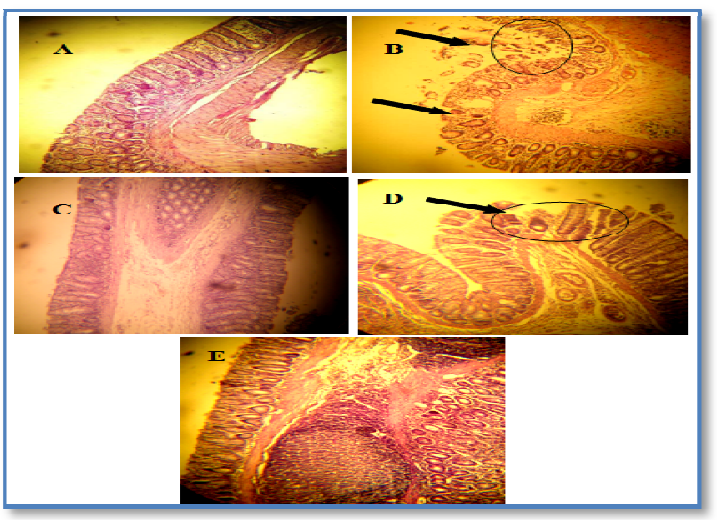

Figure2. Examples of photomicrographs of rat colonic mucosa. (A) Sham negative control rat (saline-treated), (B) Acetic acid-treated rat, (C) Fish oil-treated rat, (D) Flaxseed oil- treated rats, (E) Mesalamine treated group. Arrows and circle indicate ulcerated epithelium.

\section{EFFECT OF FISH OIL AND FLAXSEED OIL ON COLONIC HISTOLOGICAL DAMAGE INDUCED BY ACETIC ACID}

Representative histological segments of the colon in the control, AA- treatment group and in group of rats treated with FO, FSO and mesalamine enemas are shown in figure 2. AA produced a significant inflammatory response and prominent lesions in the mucosa and submucosa, with invasion of inflammatory cells mainly macrophages and neutrophils and dilation of capillaries and lymphatics,Figures $2 \mathrm{~B}$ and 3. FO treatment resulted in correction of the morphological disturbances associated with AA administration. The hisopathological score was significantly reduced from $3.7 \pm 0.2$ in the AA colitis group to $1.2 \pm 0.4$ in the FOtreatment group respectively, $(\mathrm{P}<0.01)$ (Figure $2 \mathrm{C}) . \mathrm{FSO}$, on the other hand, has no effect on tissue damage caused by AA, the histological damage score in this group was $3.2 \pm 0.5$ versus $3.7 \pm 0.2$ in AA control group and the reduction was not significant. Comparing the results of FO group with the standard drug mesalamine, the reduction in histological score was slight and insignificant $(1.2 \pm 0.4$ vs $1.5 \pm 0.6)$. The findings at microscopy were consistent with macroscopic appearance and changes in colonic weight and the weight/length ratio in the different treatment groups. These results are summarized in, figure 4 .

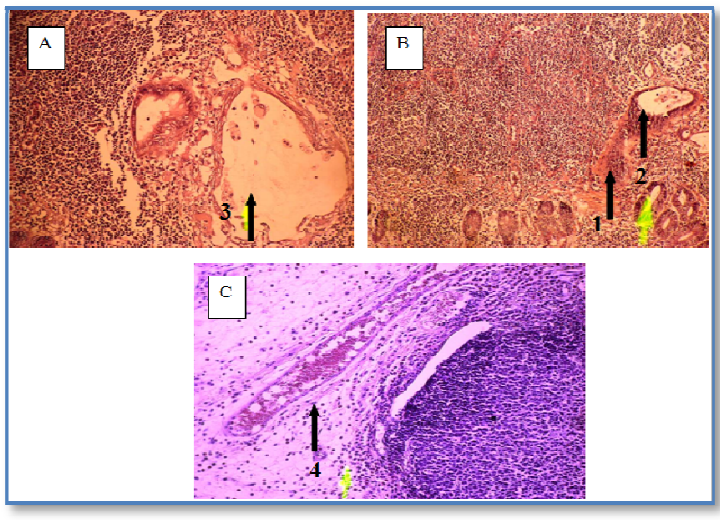

Figure3. Higher magnification of rat colon treated with acetic acid showing in A, B and C: large number of mucosal glands with hyperplastic (1) and dysplastic (2) changes with some of them lined by one or more layers and others seen completely obstructed with cystic formation (3) or papillary cystic formation. The submucosal blood vessels are severely dilated and hyperemic (4)

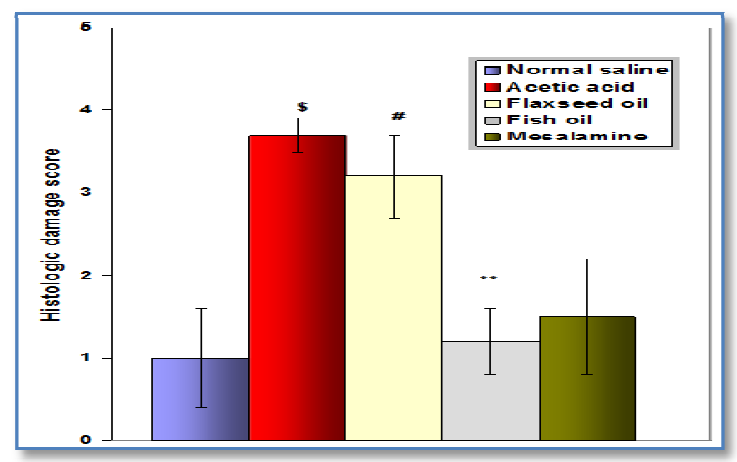

Figure 4.Effect of pretreatment with fish oil, flaxseed oil and meselamine on histological damage score in acetic acidinduced colitis model in rats. Values are mean $\pm S E M, n=6$. $\$, P<0.01$ significantly higher than normal saline (negative control) group. \#, $\mathrm{P}<0.05$ significantly higher than fish oil ; **, $\mathrm{P}<0.01$ significantly lower than acetic acid (control colitis group).

\section{DISCUSSION:}

The present study was planned to compare the protective effects of two omega-3 rich oils; the plant derived FSO and the marine derived FO on AA colitis in rats. Induction of colitis by AA is one of the standard methods to produce a model with the characteristics of human ulcerative colitis [15]. It affects the distal colon portionand induces non-transmural inflammation, massive necrosis of mucosal and submucosal layers, mucosal edema, neutrophil infiltration of mucosal and submucosal ulceration [16]. The inflammatory response initiated by AA includes activation of cyclooxygenase and lipooxygenase pathways [17]. The reason behind investigating the protective effects of FSO in this study and to compare its effects with FO, is the fact that FSO by far is the richest source of Omega-3 fatty acid alpha-linolenic acid (ALA) at roughly 57\%, six times richer than most fish oil, in n-3 [18,19]. Its 
omega6:omega3 ratio is very desirable at $0.3: 1$. The ALA is converted by metabolism in vivo into the longer chain omega 3's EPA and DHA. To our knowledge, there have been no reports on the effects of the plant derived FSO either as supplement or in the form of enema in experimental colitis. Our results have demonstrated that intracolonic administration of $4 \%$ AA caused a substantial degree of inflammation and tissue injury in the rat colon as evidenced microscopically by the increase in cellular infiltration, muscle thickness and loss of architectural structure and macroscopically by the increase in weight of colon segment compared with SC-group. The degree of tissue damage was markedly attenuated by intrarectal treatment with FO. These results are in accordance with other studies, which demonstrated that FO enema provided a protective effect against ethanol, AA and trinitrobenzene-induced colitis models in rats $[5,6]$. The protective effect was reported to be associated with a significant reduction in the levels of leukotriene B4 and myeloperoxidase activity in the colonic tissue [5]. On the other hand, we were unable to detect any protective effect of FSO in AA-induced colitis. Clinical reports [9-13] have indicated that in patients with UC, supplementation with FO was effective in reducing both the symptoms and the dose of corticosteroid required to control the disease and in reducing rate of relapse. In a case report study, FO enema produced a significant improvement in symptoms, normal-appearing colonic mucosa on colonoscopy and on histopathology [20]. The exact mechanism of omega- 3 fatty acids (n-3 FAfatty acids) involved in the attenuation of inflammations is not well understood. However, they n-3 FA are believed to reduce inflammation through reduction of the incorporation of EPA and DHA into the arachidonic acid with increased production of 3-series PGs and thromboxanes and 5-series LTs, thus reduce the inflammatory potential $[21,22]$. Interleukin- $1 \alpha$ and tumor necrosis factor production may also be reduced by the presence of n-3 FAfatty acids [23]. Thus, the propagation of inflammatory pathways is diminished. The n-3 FAfatty acids from FO have been found to inhibit cytokine and eicosanoid formation. Prolonged treatment with FO in patients with rheumatoid arthritis (RA), resulted in a significant decrease in LTB4 and TNF- 5 [24] Eicosapentaenoic acid EPA is the precursor of the PG3 series of prostaglandins and resolvins, which have anti-inflammatory effects. Docosahexaenoic acid DHA is the precursor of the docosanoids, termed 'resolvins' or 'protectins', which are analogous to the eicosanoids and have
Aisha Dugani et al potent anti-inflammatory and immune-regulatory actions $[25,26]$. Docosahexaenoic acid DHA is believed to have beneficial effects upon inflammatory disorders of the intestine and in reducing the risk of colon cancer, which may be mediated through associations with specific signaling proteins in membranes [25]. Flaxseed oil FSO ccontains $50-60 \%$ omega- 3 fatty acids in the form of ALA and this amount are roughly double that contained in FO. However, the results obtained in this study using FSO in the form of enema showed that it didn't provide any significant protective effect against AA-inducing colonic injury. This may be attributed to the fact that ALA present in FSO is a precursor and has to be converted in vivo into two major metabolites; the long chain omega-3 fatty acids, eicosapentaenoic acid epa and docasahexaenoic acid dha [18], such conversion require systemic and not local treatment. Therefore, further investigations are needed to explore the effect of changing the route of administration of FSO using oral feeding.

\section{CONCLUSION:}

The results presented in this article reflect that FO but not FSO is beneficial in the prevention of UC induced by AA when given in the form of enema. This data reinforce the previous reports on the beneficial effects of omega-3 rich compounds on IBD. However, we could not completely rule out any beneficial effects of FSO in UC until further investigations using the oral route of administration are completed.

\section{A CKNOWLEDGMENT AND SOURCE FUNDING:}

This research received no specific grant from any funding agency in the public, commercial, or notfor-profit sectors.

\section{REFERENCES:}

1) Head $\mathrm{k}$ and jurenka j. ulcerative colitis: inflammatory bowel disease part i: ulcerative colitis-pathophysiology and conventional and alternative treatment options. altern med rev; 8: 247-283, 2003.

2) podolsky $\mathrm{dk}$. inflammaotory bowel disease. $n$ engl j med; 347: 417-429, 2002.

3) scumert $r$, towner $j$, zipser $r d$. role of eicosanoids in human and experimental colitis. dig dsi sci; 33: 58-64, 1988.

4) ardizzone yuceyar $h$, saruc $m$, ozutmiz, et al.immunomodulators for all patients with inflammatory bowel disease? therap adv gastroenterol;3(1):31-42, 2010. 
5) yuceyar $h$, saruc $m$, ozutmiz, et al. the protective effect of fish oil enema in acetic acid and ethanol induced colitis in rats. the turkish journal of gastroenterology 2: 155-161, 2000.

6) yuceyar h, ozutemiz o, huseyinov a, saruç $\mathrm{m}$, alkanat $\mathrm{m}$, bor $\mathrm{s}$, coker i, batur $\mathrm{y}$. is administration of $n-3$ fatty acids by mucosal enema protective against trinitrobenzene-induced colitis in rats? prostaglandins leukot essent fatty acids. dec; 61(6): 339-45, 1999.

7) garg $\mathrm{ml}$, thomson $\mathrm{ab}$, cladinin t. fish oilenriched diet is mucosal protective against acetic acid-induced colitis in rats. can $\mathrm{j}$ physiol pharmacol; 69: 480-7, 1991.

8) stenson wf, cort $d$, rodgers $j$, et al. dietary supplementation with fish oil in ulcerative colitis. ann intern med; 116: 609-14, 1992.

9) mccall $\mathrm{t}$, o'leary $\mathrm{d}$, bloomfield $\mathrm{j}$, o'moráin c. therapeutic potential of fish oil in the treatment of ulcerative colitis.aliment pharmacol ther; 3(5): 415-24, 1989.

10) salomon $p$, kornbluth a, janowitz $h$. treatment of ulcerative colitis with fish oil n-3omega-fatty acid: an open trial. $\mathrm{j}$ clin gastroenterol; 12(2): 157-61, 1990.

11) belluzzi a, brignola $\mathrm{c}$, campieri $\mathrm{m}$, pera $\mathrm{a}$, boschi s, miglioli m.effect of an enteric-coated fish-oil preparation on relapses in crohn's disease. $\mathrm{n}$ engl j med;334(24): 1557-60, 1996.

12) turner $d$, steinhart a, griffiths a. omega 3 fatty acids (fish oil) for maintenance of remission in ulcerative colitis. cochrane database syst rev; (3): cd006443, 2007.

13) hawthorne ab, daneshmend tk, hawkey cj, et al. treatment of ulcerative colitis with fish oil supplementation: a prospective 12 month randomized controlled trial. gut 1992; 33: 922-8.

14) gulec $b$, yasar $m$, yildiz $s$, oter $s$, akay $c$, deveci s, sen d. effect of hyperbaric oxygen on experimental acute distal colitis. physiol res 53: 493-500, 2004.

15) fabia $r$, willén $r$, ar'rajab a, andersson $r$, ahrén $\mathrm{b}$, bengmark $\mathrm{s}$. acetic acid-induced colitis in the rat: a reproducible experimental model for acute ulcerative colitis. eur surg res.; 24(4): 211-25, 1992.

16) nakhai 1, mohammadirad a, yasa $n$, minaie $b$, nikfar s, ghazanfari g, et al. benefits of zataria multiflora boiss in experimental model of mouse
Aisha Dugani et al inflammatory bowel disease. ecam; 4:43-50, 2007.

17) sharon $p$, stenson wf. metabolism of arachidonic acid in acetic acid colitis in rats. gastroenteroloogy; 88:55-63, 1985.

18) cunnane sc, ganguli s, menard $\mathrm{c}$, liede ac, hamadeh mj, chen zyliede ac, hamadeh mj, chen zy, et al. high $\alpha$-linolenic acid flaxseed (linum usitatissimum): some nutritional properties in humans. br j nutr; 49:443-453, 1993.

19) thomas bartram (1998). bartram's encyclopedia of herbal medicine. pp. 271.

20) tatar e and das $k$. improvement in ulcerative colitis symptoms after use of fish oil enema. gastroenterology and hepathology.3:786-788, 2007.

21) belluzzi a. $n-3$ fatty acids for the treatment of inflammatory bowel diseases. proceed nutr soc.;61:391-395, 2002.

22) sharon $p$, stenson wf. enhanced synthesis of leukotriene $\mathrm{b} 4$ by colonic mucosal inflammatory bowel disease. gastroenterology.;86:453-460, 1984.

23) endres s, ghorbani r, kelly ve, georgilis k, lonnemann g, van der meer jw, et al. the effect of dietary supplementation with n-3 fatty acids on the synthesis of interleukin-1 and tumor necrosis factor by mononuclear cells. $\mathrm{n}$ engl med.:320:265-270, 1989.

24) adam $o$, beringer $c$, kless $t$, lemmen $c$, adam a, wiseman $\mathrm{m}$, et al. anti-inflammatory effects of low arachidonic acid diet and fish oil in patients with rheumatoid arthritis. rheumatol. int; 23: 2736, 2003.

25) christio ww. fatty acids:methylene interurrupted double bonds . structure, occurance and biochemistry (accessed on august, 2009).

26) kohli, p. and levy, b.d. resolvins and protectins: mediating solutions to inflammation. brit. j. pharm; 158, 960-971, 2009. 27) harris, w. s. fish oils and plasma lipid and lipoprotein metabolism in humans: a critical review. j. lipid res. 30:785-807, 1989.

28) bonna kh, bjerve ks, straume b, gram it, thelle, d. effects of eicosapentaenoic and docosahexaenoic acids on blood pressure in hypertension. a population-based intervention trial from the tromso study. n. engl. j. med. 322: 795-801, 1990. 
29) Beck sa, smith kl, tisdale mj. anticachectic and antitumor effect of eicosapentaenoic acid and its effect on protein turnover. cancer res. 51: 6089-6093, 1991.

30) robinson dr, xu 11, tatenom s, guo $m$, colvin rb. suppression of autoimmune disease by dietary n-3 fatty acids. j. lipid res. 34: 1435-1444, 1993. 31) dugani a, auzzi a, naas f, megwez s. effects of the oil and mucilage from flaxseed (linum usitatissimum) on gastric lesions induced by ethanol in rats. libyan j med, 3: d166-169, 2008 